International Journal of Innovative Engineering Applications

Journal homepage: https://dergipark.org.tr/ijiea

\title{
THE SEASONAL BEHAVIOR OF THE CHARACTERISTIC WAVE IN LOW LATITUDES
}

\author{
Kadri Kurt ${ }^{* 1}$ \\ ${ }^{I}$ Fırat Üniversitesi, Fen Fakültesi, Fizik Bölümü, Elazığ, Türkiye
}

\begin{abstract}
Original scientific paper

This study aims to investigate the magnitude of polarization of characteristic wave $\left(D_{z}=0\right)$, for all seasons by using the real geometry of the Earth's magnetic field for the selected altitudes $(390,410,450,500,550$ and $600 \mathrm{Km})$ in the equatorial anomaly region at low latitudes $\left(-30^{\circ} \mathrm{S}\right.$ and $\left.30^{\circ} \mathrm{N}\right)$. The part of imaginary of the characteristic wave having a complex structure in latitudes where equatorial anomaly occurs it has a dramatically resemblance to the change of electron density, and the real part has been similarity showing with the change with latitude of the refractive index for all seasons for 12.00-24.00LT.
\end{abstract}

Keywords: Ionosphere, Characteristic wave, Equatorial Anomaly

\section{Introduction}

So far, many important studies have been done and continue to be done in order to know the chemical and physical structure of the ionosphere and recognize the structure of the ionosphere. In addition, more advanced studies are needed in terms of communication and technology regarding the interaction of the ionosphere with the electromagnetic wave, and these studies are increasingly continuing [1-18]. In this sense, the study of wave polarization is only one of them. As a concept, polarization is used in physics in two basic terms. The first of them is volume polarization, and the other is the polarization of wave $[8,10-12]$. It is the wave that does not change the polarization direction when the characteristic wave propagates any medium. When there is any possibility of confusion, these shall refer to two magnitudes as "the volume-polarization and the wavepolarization" respectively. It is interesting to note that the two meanings, now thought of quite differently, originated in the same idea that of producing opposite polarity at opposite ends of line. The relation to the volume polarization is obvious. In relation to wavepolarization, the term arose when it was thought that light was the corpuscles in nature, and to explain the facts of double refraction it was suggested that the corpuscles acquired a polarity along a certain direction, known as the direction of polarization. But these two polarities are not independent from each other. From a detailed analysis of the response of the charge carriers to the oscillating $\mathrm{E}$ and $\mathrm{B}$ fields, one can deduce a linear relationship between the electric field $\mathrm{E}$ of wave and the polarization [9-11].
The behavior of the F2- region in the ionosphere is different at low latitudes [1-6]. Sometimes, the electron density at midnight is greater than the electron density at noon because of ionization is scattered throughout the magnetic field of the Earth. This distribution affects the latitude distribution of ionization and the daily change of electron density formed in electromagnetic drift. The electron density is seen, peaks between $15^{0}-20^{0}$ north and $00-5^{0}$ south, in cavities (magnetic equator) $5^{0}-10^{0}$ North. The behavior of electron density at $\mathrm{F} 2$ region is called as the equatorial anomaly in ionosphere plasma [4,5,7].

Many researchers have done extensive research on the physical and chemical processes in the ionosphere and the behavior of the electromagnetic wave in the ionosphere, especially propagation, reflection and refractive index like many more subjects [1-7]. However, there are limited studies on the polarization of the electromagnetic wave in ionospheric plasma [8-14]. We have focused on this issue for some special conditions in ionospheric plasma. Because, polarization is a measure of the behavior of the electromagnetic wave in a medium and the response of the medium.

In this study, we investigated the relationship between the magnitude polarization of a characteristic wave and the equatorial anomaly for different heights of the ionosphere F2-region.

\section{Volume Polarization Tensor for Cold Ionosphere Plasma}

We obtained the volume polarization tensor for the cold plasma conditions in ionospheric plasma and compared the magnitudes of volume polarization the 
ambient magnetic field in northern hemisphere is given by [3-6];

$\mathbf{B}=B_{x} \mathbf{a}_{x}+B_{y} \mathbf{a}_{y}+B_{z} \mathbf{a}_{z}$

Where $\mathrm{B}_{\mathrm{x}}=\mathrm{B}_{0} \operatorname{CosI}$ Sind, $\mathrm{B}_{\mathrm{y}}=\mathrm{B}_{0} \operatorname{Cos} I \operatorname{Cos} d$ and $\mathrm{B}_{\mathrm{z}}=$ $\mathrm{B}_{0}$ SinI. I and $\mathrm{d}$ are the magnetic dip and the magnetic declination angles, respectively. The force acting on electron in the cold plasma is given by

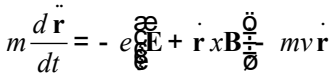

In which $\mathbf{r}, \mathbf{B}, \mathbf{E}$ and $v$ (=electron-ion+electron-notr collisions frequency) are position vector (r), Earth magnetic field, electric field and electron mass (m) and elecrtoncollsion frequency, respectively. In here, all of fields change like $e^{i}(\omega t-k . r)$. The movement of an electron from a point $\mathrm{A}$ to point $\mathrm{B}$ is equivalent to leaving the original electron undisturbed at $\mathrm{A}$ and adding a dipole [15].

If there are $\mathrm{N}$ electron per unit volume, and if all move through equal distance $\mathbf{r}$, equivalent dipole moment, which is the polarization is given by [11-15].

$\mathbf{P}=N e \mathbf{r}=e_{0} \chi \mathbf{E}$

The movements of the electrons and the polarization $\mathbf{P}$ is produced by the electric field of wave $\mathbf{E}$. If there is no applied magnetic field, and then $\mathbf{P}$ and $\mathbf{E}$ are parallel, otherwise $\mathbf{P}$ and $\mathbf{E}$ are not parallel. If we use Eqs. (1-3) together, Volume polarization for northern hemisphere is obtained by

$$
\begin{aligned}
& \left(i \omega v-\omega^{2}\right) P_{x}-i \omega \omega_{c z} P_{y}+i \omega \omega_{c y} P_{z}=\frac{N e^{2}}{m} E_{x} \\
& \left(i \omega v-\omega^{2}\right) P_{y}+i \omega \omega_{c z} P_{x}-i \omega \omega_{c x} P_{z}=\frac{N e^{2}}{m} E_{y} \\
& \left(i \omega v-\omega^{2}\right) P_{z}-i \omega \omega_{c y} P_{x}+i \omega \omega_{c x} P_{y}=\frac{N e^{2}}{m} E_{z}
\end{aligned}
$$

from here,

$$
\begin{aligned}
& P_{x}=\frac{a\left[\left(i v \omega-\omega^{2}\right)^{2}+\omega^{2} \omega^{2} c\right]}{A+i B} E_{x}+\frac{a\left[i \omega \omega_{c z}\left(i v \omega-\omega^{2}\right)^{2}-\omega^{2} \omega_{c x} \omega_{c y}\right]}{A+i B} E_{y} \\
& +\frac{a\left[-i \omega \omega_{c y}\left(i v \omega-\omega^{2}\right)^{2}-\omega^{2} \omega_{c x} \omega_{c z}\right]}{A+i B} E_{z} \\
& P_{y}=\frac{-a\left[i \omega \omega_{c z}\left(i v \omega-\omega^{2}\right)+\omega^{2} \omega_{c x} \omega_{c y}\right]}{A+i B} E_{x}+\frac{a\left[\left(i v \omega-\omega^{2}\right)^{2}-\omega^{2} \omega_{c y}^{2}\right]}{A+i B} E_{y} \\
& +\frac{a\left[i \omega \omega_{c x}\left(i v \omega-\omega^{2}\right)^{2}-\omega^{2} \omega_{c y} \omega_{c z}\right]}{A+i B} E_{z} \\
& P_{z}=\frac{a\left[i \omega \omega_{c y}\left(i v \omega-\omega^{2}\right)-\omega^{2} \omega_{c x} \omega_{c z}\right]}{A+i B} E_{x}+\frac{a\left[-i \omega \omega_{c x}\left(i v \omega-\omega^{2}\right)^{2}-\omega^{2} \omega_{c z} \omega_{c y}\right]}{A+i B} E_{y} \\
& +\frac{a\left[\left(i v \omega-\omega^{2}\right)^{2}-\omega^{2} \omega_{c z}^{2}\right]}{A+i B} E_{z}
\end{aligned}
$$

If all the polarization coefficients are rearranged both real and imaginary, $\mathrm{P}_{\mathrm{x}}, \mathrm{P}_{\mathrm{y}}$ and $\mathrm{P}_{\mathrm{z}}$ are obtained as.

$$
P_{x}=\left(\chi_{11 R}-i \chi_{11 s}\right) E x+\left(\chi_{12 R}+i \chi_{12 s}\right) E_{y}+\left(\chi_{13 R}+i \chi_{13 s}\right) E_{z}
$$

$$
\begin{aligned}
& P_{y}=\left(\chi_{21 R}+i \chi_{21 s}\right) E_{x}+\left(\chi_{22 R}-i \chi_{22 s}\right) E_{y}+\left(\chi_{23 R}-i \chi_{23 s}\right) E_{z} \\
& P_{z}=\left(\chi_{31 R}-i \chi_{31 s}\right) E x+\left(\chi_{32 R}+i \chi_{32 s}\right) E_{y}+\left(\chi_{33 R}-i \chi_{33 s}\right) E_{z}
\end{aligned}
$$

Volume-polarization tensor could be obtained depending the permittivity coefficient $(\chi)$ of ionospheric plasma as follow

\section{The Polarization of Characteristic Wave in The Cold lonospheric Plasma}

The electric flux (D) of any medium is given by well-known equation as follow [8-15].

$\mathbf{D}=\varepsilon_{0} \mathbf{E}+\mathbf{P}$

The electric flux into propagation direction of the charteristic wave cannot be occurred. Due to this, $D_{z}=0$, when this condition is used into eq. (12) and substitute into Eqs. (10-11). The coefficiens of polarization are obtained by

$$
\begin{aligned}
P_{x} & =\left(\mu_{R}+i \mu_{S}\right) E_{x}+\left(\sigma_{R}+i \sigma_{S}\right) E_{y} \\
P_{y} & =\left(\rho_{R}+i \rho_{S}\right) E_{x}+\left(W_{R}+i W_{S}\right) E_{y} \\
\frac{P_{x}}{P_{y}} & =\frac{E_{x}}{E_{y}}
\end{aligned}
$$

when eqs. (15-17) are used together; the equation of chracteristic wave is as follow.

$$
\left(\frac{E_{x}}{E_{y}}\right)_{1,2}=\frac{-\left(K_{R}+i K_{S}\right)}{2\left(\rho_{R}^{2}+\rho_{S}^{2}\right)} \mp \frac{\sqrt{K_{1 R}+i K_{1 S}}}{2\left(\rho_{R}^{2}+\rho_{S}^{2}\right)}
$$

We could write for the expression in square root after mathematical manipulation as follow.

$$
\sqrt{K_{1 R}+i K_{1 S}}=\sqrt{K_{1 R}^{2}+K_{1 S}^{2}}\left[\cos \left(\frac{\theta+2 k \pi}{n}\right)+i \sin \left(\frac{\theta+2 k \pi}{n}\right)\right]
$$

in which $\mathrm{n}=2, \mathrm{k}=0,1$

\section{Numerical Analysis and Results}

In this study, the wave polarization for the characteristic wave was calculated as seasonal the latitudes by using Eq. (17), at hour 12.00 and 24.00 and as local time for 1990 year. The ionospheric parameters used for calculation were obtained by using the IRI model, according to the accepted conditions. We investigated the seasonal change of eqn. (17) with respect to latitude for 12.00 and $24.00 \mathrm{LT}$ at $390,410,450,500$, 550 and $600 \mathrm{~km}$ altitudes (these heights are the altitudes at which equatorial anomaly occurs at F2-Region and low latitudes in ionospheric plasma) for the characteristic wave in ionospheric plasma. The most important feature of the characteristic wave; as wave travel in any medium, the polarization direction of wave cannot change. Besides, there is no induced magnetic polarization corresponding to electric polarization and in the direction of the wave's progress doesn't occur the electric flux. For 
corresponded conditions in Figure 1, the magnitude of both the real and the imaginary part of the wave polarization are given for latitudes at $12.00-24.00 \mathrm{LT}$ as seasonal. Looking at all the Figures (1-4), we can make a general comment on the magnitude of the real part of wave polarization, for all seasons. They are the same trends for all seasons and almost equal in magnitude. Their variation decreases exponentially, peaking between $-10^{\circ}$ and $-20^{\circ}$ at low latitudes at both day and night for all season and the refractive index is order of magnitude (Figure 1-4). But the behaviour of the magnitude of the imaginary part of the characteristic wave at low latitudes is interesting for all seasons. The magnitude of the imaginary part of the characteristic wave is given at Figure 1 for March 21. According to this, for the heights of $600,550,550 \mathrm{~km}$ the magnitudes of polarization (for imaginary part) are sharply decreasing very close to the equator, making a striking peak at about $-5^{0}$ south. However, for other heights $(390,410$ and 450 $\mathrm{Km})$ there is a maximum increase $10^{0}$ south. Its numerical values are larger at night compared to daytime and has a peak at $10^{\circ} \mathrm{S}$ for all season at $24.00 \mathrm{LT}$.

For June 21, the magnitude of the imaginary part of the characteristic wave is given in Figure 2 for low latitudes at 12.00 and 24.00 LT. Accordingly, in both cases, a cavity occurred approximately at $0-10^{0} \mathrm{~S}$ and had the smallest value and continues to increase after this latitude. For imaginary parts, similar situations are observed in other seasons for 12.00 and 24.00LT (Sempt.23, December 21). The behavior of the magnitude of the imaginary part of the characteristic wave at low latitudes is generally similar to that of the electron density. However, this adjustment is observed more sharply at lower heights. The changes of the imaginary part with latitude at $12.00 \mathrm{LT}$ are very similar in terms of both trend and size for March-December and June-September for $450,500,550$ and $600 \mathrm{Km}$. The trend has sharper ups and downs for 390 and $400 \mathrm{Km}$. These are compatible with the literature [1,7-8].

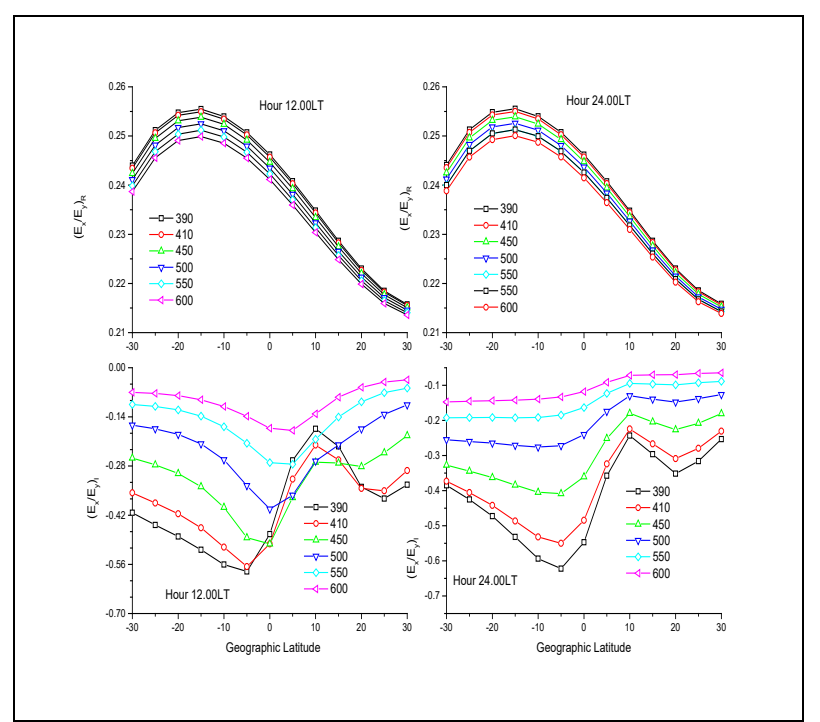

Figure 1. The change with latitude imaginary of parts of the polarization wave (12.00 and 24.00LT; March 21).

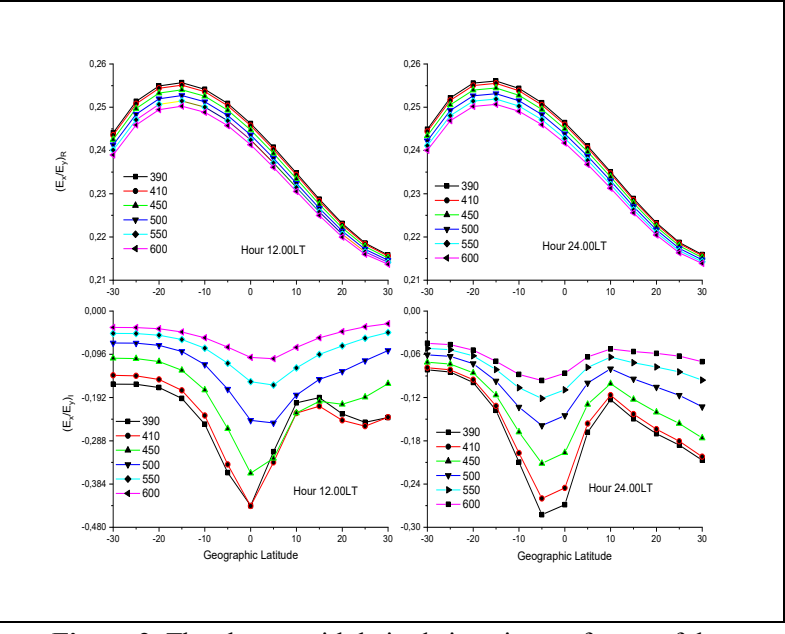

Figure 2. The change with latitude imaginary of parts of the polarization wave (12.00 and 24.00LT; June 21)

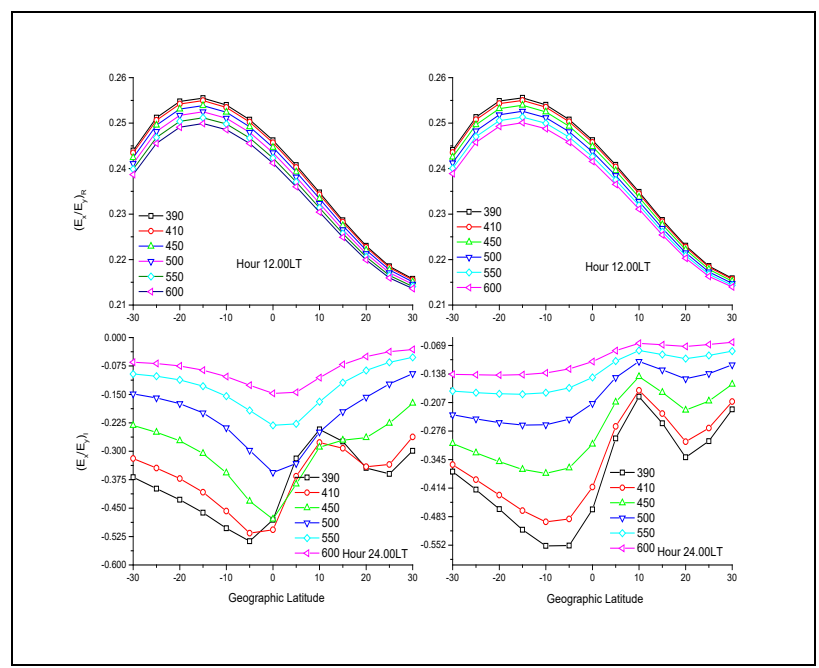

Figure 3. The change with latitude imaginary of parts of the polarization wave (12.00 and 24.00LT; Sempt. 23).

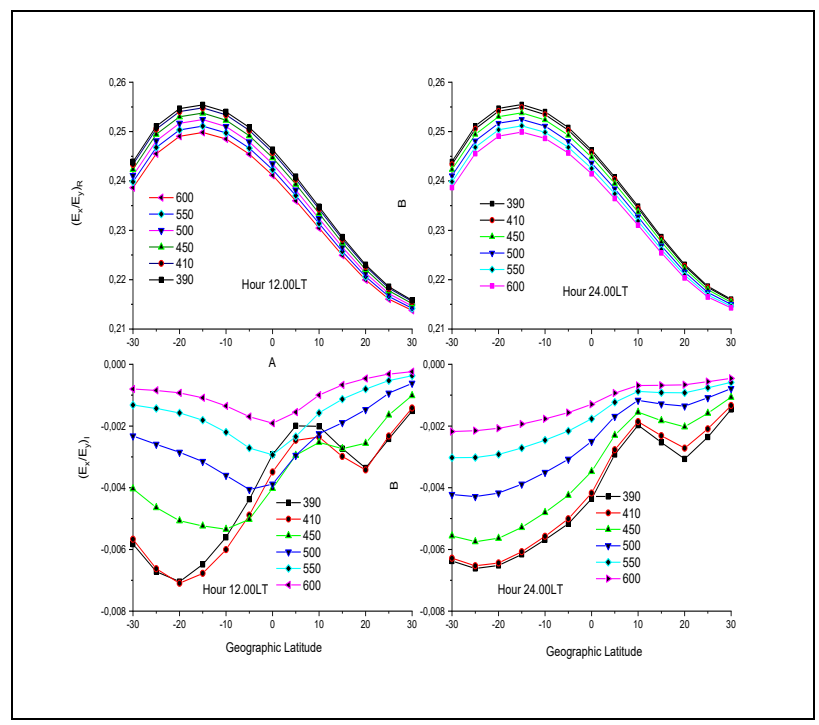

Figure 4. The change with latitude imaginary of parts of the polarization wave (12.00 and 24.00LT; December 21). 


\section{Conclusions}

In this study it has been investigated the magnitude of polarization of characteristic wave $\left(D_{z}=0\right)$, for all seasons by using the real geometry of the Earth's magnetic field in low latitudes for ionospheric plasma with respect to Eq. (17). The results obtained are truly surprising. As a matter of fact, if the earth's magnetic field is taken into account, polarization is ecliptic and the electric field vector does not sweep a circle. Theoretically, this is not surprising. However, it is interesting to see that both the real part and the imaginary part have great values mid-latitudes. For the accepted conditions, the conclusion we infer from all these data; The real part of the magnitude of polarization of the characteristic wave is approximately similar for all seasons and very close as magnitude refractive index order of it $[4,11]$. But the imaginary part is actually very close to the change of electron density at low latitudes. Moreover, magnitudes in summer are larger than in winter, and central cavities appear on the magnetic dip equator "approximately between $-10^{\circ} \mathrm{S} ; 10^{\circ} \mathrm{N}$ ".

\section{References}

[1] Ünal, İ., Şenalp, E. T., Yeşil, A., Tulunay, E., \& Tulunay, Y. (2011). Performance of IRI-based ionospheric critical frequency calculations with reference to forecasting. Radio Science, 46(01), 1-10.

[2] Yeşil, A., \& Ünal, İ. (2011). Electromagnetic wave propagation in ionospheric plasma. Behaviour of Electromagnetic Waves in Different Media and Structures, 189.

[3] Yeşil, A., \& Sağır, S. (2019). Updating Conductivity Tensor of Cold and Warm Plasma for Equatorial Ionosphere F2-Region in The Northern Hemisphere. Iranian Journal of Science and Technology, Transactions A: Science, 43(1), 315-320.

[4] Sağır, S., \& Yeșil, A. (2018). The Relation Between the Refractive Index of the Equatorial Ionospheric F2 Region and Long-Term Solar Indices. Wireless Personal Communications, 102(1), 31-40.
[5] Yesil, A., \& Kurt, K. (2018). Calculation of electric field strength in the ionospheric F-region. Thermal Science, 22(Suppl. 1), 159-164.

[6] Timoçin, E., Yeşil, A., \& Ünal, İ. (2014). The effect of the geomagnetic activity to the hourly variations of ionospheric foF2 values at low latitudes. Arabian Journal of Geosciences, 7(10), 4437-4442.

[7] Swanson, D. G. (2012). Plasma waves. Elsevier.

[8] Whitten, R.C., Poppoff, I.G. (1971). Fundamentals of Aeoronmy, John Willey and Sons, New York.

[9] Budden, K.G. (1988). The Propagation of Radio Waves, Cambridge University Press, Cambridge.

[10] Budden, K. G., \& Stott, G. F. (1980). Rays in magnetoionic theory-II. Journal of Atmospheric and Terrestrial Physics, 42(9-10), 791-800.

[11] Richard, F. (2014) The physics of Plasma, CRC press, New York, 50-140.

[12] Rishbeth, H. (1973). Physics and chemistry of the ionosphere. Contemporary Physics, 14(3), 229-249.

[13] Rishbeth, H., \& Garriott, O. K. (1969). Introduction to ionospheric physics. Introduction to ionospheric physics.

[14] Ratcliffe, J.A. (1959). The magneto-ionic Theory and Its applications to the ionosphere, Cambridge at the University Press, London.

[15] Sağir, S., Yaşar, M., \& Atici, R. (2019). The Relationship between Dst, IMF-Bz and Collision Parameters for $\mathrm{O}++\mathrm{N}$ $2 \rightarrow \mathrm{NO}++\mathrm{N}$ Reactive Scattering in the Ionosphere. Geomagnetism and Aeronomy, 59(8), 1003-1008.

[16] Yaşar, M. (2021). The solar eclipse effect on diffusion processes of $\mathrm{O}++\mathrm{O} 2 \rightarrow \mathrm{O} 2++\mathrm{O}$ reaction for the upper ionosphere over Kharkov. Thermal Science, (00), 7-7.

[17] Yasar, M. (2021). The Change Of Diffusion Processes For $\mathrm{O}++\mathrm{N} 2 \rightarrow \mathrm{No}++\mathrm{N}$ Reaction In The Ionospheric F Region Durıng The Solar Eclıpse Over Kharkov, Thermal Science, 25 (1), 51-56.

[18] Yaşar, M., Atıc1, R., \& Sağır, S. (2018). The change of the collision parameters of ' $\mathrm{O}^{+}+\mathrm{N}_{2} \rightarrow \mathrm{NO}^{+}+\mathrm{N}$ ' reaction according to geomagnetic activity days in the ionosphere, MSU J. Sci., 6, 529-532. 